\title{
Diagnosis and Therapeutic Management of Theileriosis in Cattle Calves
}

\section{Savita ${ }^{1}$, Dhuria $\mathrm{D}^{2 *}$ and Nayak $\mathrm{TC}^{1}$}

${ }^{1}$ Department of Clinical Veterinary Medicine, Ethics and Jurisprudence, India

${ }^{2}$ Department of Clinical Veterinary Medicine, Ethics and Jurisprudence, College of Veterinary and Animal Science, India

*Corresponding author: Deepika Dhuria, Department of Clinical Veterinary Medicine Ethics and Jurisprudence, College of Veterinary and Animal Science, RAJUVAS, Bikaner-334 001, Rajasthan, India, Email: drnandalsavita@ gmail.com

\section{Abstract}

The present investigation was carried out among ten cross-bred Cattle calves of age between 1-5 months which were presented to Veterinary Clinical Complex of College of Veterinary and Animal Science, Bikaner with the history of anorexia, high fever, diarrhoea and tick infestation. Upon clinical examination pale mucous membrane, enlarged prescapular lymph nodes and increase in respiration rate and pulse rate were found. Blood samples were collected from jugular vein in vacutainers containing EDTA for haemogram. Microscopic examination of Giemsa stained thin blood smears revealed piroplasms in erythrocytes and lymph node aspirate smears revealed presence of schizonts (Koch's blue bodies) in lymphocytes. Upon haematological examination it revealed that the decrease in haemoglobin, total erythrocyte count, packed cell volume and lymphocytes, however increase in total leukocyte count and neutrophils. After confirmation of Theileriosis in calves Buparvaquone was administered at the dose rate of $1 \mathrm{ml} / 20 \mathrm{~kg}$ body weight via intramuscular route. Eight calves were very well responded to the treatment but two calves were eventually died due to severe anaemia.

Keywords: Theileriosis; Piroplasms; Schizonts; Buparvaquone

\section{Introduction}

Tropical theileriosis (Theileria annulata infection); is a tick-borne haemoprotozoan disease of mainly cross-bred cattle calves which is transmitted by Hyalomma anatolicum anatolicum tick. Tropical Theileriosis is an economically important disease of cattle in tropical and subtropical regions [1] that stretch out from the Mediterranean coastal regions to Indian subcontinent and China [2]. It usually occurs in late spring and early summer season in animals [3]. In $T$. annulata infection common clinical symptoms are weakness, weight loss, anorexia, high fever, petechial haemorrhages on conjunctiva, swollen lymph nodes (parotid, prescapular and prefemoral) and anaemia and in later stage icterus, dehydration and blood in faeces are the occasional clinical symptoms [4-6].

Conventional method of diagnosis of tropical theileriosis largely depends on examination of Giemsa stained thin blood and lymph node aspirate smears [7]. Buparvaquone is a second generation hydroxynaphthoquinone compound and drug of choice for tropical Theileriosis $[8,9]$. This paper presents direct microscopic detection of the piroplasms and schizonts of Theileria annulata in blood smears and lymph 
node biopsy smears (easy, convenient and 'gold standard' diagnostic test) from the cattle calves which shown signs of high fever and enlargement of lymph nodes and treatment of calves with Buparvaquone which is a promising drug for the treatment of theileriosis in animals.

\section{Materials and Methods}

The present investigation was carried out among ten cross-bred Cattle calves of age between 1-5 months which were presented to the Veterinary Clinical Complex, College of Veterinary and Animal Science, Bikaner with the history of anorexia, high fever, diarrhoea and tick infestation. Upon clinical examination pale mucous membrane, enlarged prescapular lymph nodes and increase in respiration rate and pulse rate were found.

\section{Diagnosis}

Blood samples were collected from jugular vein in vacutainers containing EDTA for haemogram. Giemsa stained thin blood smears were also examined for piroplasms and lymph node biopsy smear for schizonts. Faecal samples were also collected for examination of parasitic infestation.

\section{Treatment}

Buparvaquone (inj. Zubion) was administered in each calf at the dose rate of $1 \mathrm{ml}$ per $20 \mathrm{~kg}$ body weight via intramuscular route. The calves were clinically examined at $3^{\text {th }}$ day of treatment to assess the efficacy of treatment.

\section{Results and Discussion}

The haematological examination of Theileria affected calves revealed that the decrease in haemoglobin, total erythrocyte count, packed cell volume and lymphocytes, however increase in total leukocyte count and neutrophils (Table 1). The findings were in accordance with Al-Emarah, et al., Al-Hosary, et al., Modi, et al. and Goyal, et al. [10-12].

\begin{tabular}{|c|c|}
\hline Parameters & Mean Value \\
\hline $\mathrm{Hb}(\mathrm{g} / \mathrm{dl})$ & 5.8 \\
\hline $\mathrm{RBC}\left(\times 10^{6} / \mu \mathrm{L}\right)$ & 4.2 \\
\hline $\mathrm{WBC}\left(\left(\times 10^{3} / \mu \mathrm{L}\right)\right.$ & 9.5 \\
\hline Lymphocytes $(\%)$ & 46 \\
\hline Neutrophils (\%) & 50 \\
\hline Eosinophils (\%) & 2 \\
\hline Monocytes (\%) & 1 \\
\hline PCV (\%) & 21.5 \\
\hline
\end{tabular}

Table 1: Mean Haematological values in Theileria affected Cattle calves.
Microscopic examination of Giemsa stained thin blood smears revealed piroplasms in erythrocytes and lymph node aspirate smears revealed presence of schizonts (Koch's blue bodies) in lymphocytes. This conventional method of diagnosis of Theileriosis was also reported by Padhiyar, et al. [13], Sharieff, et al. [7]. Microscopic examination of faecal samples did not reveal any parasitic egg or oocyst.

After confirmation of Theileriosis in calves Buparvaquone was administered in each calf at the dose rate of $1 \mathrm{ml} / 20 \mathrm{~kg}$ body weight by intramuscular route (repeat after 48 hours only two calves which did not shown any improvement), along with oral administration of diaroak powder 10 gram $\mathrm{BD}, \mathrm{SOS}$. Usage of Buparvaquone for treatment of Theileriosis in cattle calves at a dose rate of $1 \mathrm{ml} / 20 \mathrm{~kg}$ body weight was also reported by Zahid, et al. [14], Arindam Samanta, et al. [8], Singh, et al. [9]. Kumar, et al. [15] stated that Buparvaquone is a promising compound for the therapy and prophylaxis of all forms of theileriosis in animals. Singh, et al. [16] reported that efficacy of oxytetracycline in treatment of theileriosis in cows is less as compare to buparvaquone as all animals were responded to the treatment with buparvaquone.

Effect of therapy was observed in eight calves, which revealed a marked clinical improvement in appetite, body temperature and diaorrhoea subsided gradually within 3 days [8], however two calves were eventually died on first day of treatment which can be due to severe anaemia [17].

\section{Conclusion}

It may be conclude that Theileriosis inflicts economic burden on cattle breeders in terms of mortality and morbidity which reflects severe economic losses and that elevates the poverty level. Thus apart from prompt diagnosis and therapeutic management, immunoprophylaxis with in vitro attenuated schizontal cell culture vaccine which is available with trade name of Rakshavac $\mathrm{T}$ is essential to prevent infection in highly valued animals.

\section{References}

1. Hasanpour A, Sabegh YG, Sadeghi Nasab A (2013) Assessment of serum antioxidant enzymes activity in cattle suffering from theileriosis. European Journal of Experimental Biology 3(1): 493-496.

2. Dolan TT, Injairu R, Gisemba F, Mania JN, Mbadi G, et al. (1992) A clinical trial of buparvaquinone in the treatment of East-Coast fever. Veterinary Record 130: 536-538.

3. Srivastava RYN (1989) Epidemiology and control of arthropod borne protozoan diseases of livestock and birds. Compendium of summer, institute on Arthropod 


\section{International Journal of Zoology and Animal Biology}

borne diseases of domestic animals and birds and their control, Veterinary College Ranchi pp: 97-106.

4. Bakheit MA, Schnittger J, Salih DA, Boguslawski K, Beyer D, et al. (2004) Application of the recombinant Theileria annulata surface protein in an indirect ELISA for the diagnosis of tropical theileriosis. Parasitology Resarch 92(4): 299-302.

5. Radostits OM, Gay CC, Hinchcliff KW, Constable PD (2007) Veterinary Medicine. $10^{\text {th }}$ (Edn.), W.B. Saunders Company Ltd. Philadelphia.

6. Goyal P (2017) Studies on some aspects of bovine tropical theileriosis in cattle-calves. M.V.Sc. Thesis submitted to Rajasthan University of Veterinary and Animal Sciences, Bikaner, Rajasthan, India.

7. Sharieff OE, Hassan SM, Salih DA, El Hussein AM (2017) Attenuation of two field isolates of Theileria annulata and their potential as vaccine candidates against tropical Theileriosis in the Sudan. International Journal of Scientific Research in Science, Engineering and Technology (3)1: 54-58.

8. Samanta A, Dutta BA (2012) A case report of tropical theileriosis and its treatment. Exploratory Animal and Medical Research 2(1): 81-84.

9. Singh N, Mehta H, Bagherwal RK (2012) Efficacy of some chemotherapeutic drugs against Theileria annulata infection in crossbred calves. Indian Journal of Veterinary Research 21(1): 73-76.

10. Al-Emarah GYA, Khudor MH, Daham HR (2012) Clinical, haematological and biochemical study to cattle naturally infected with Theileria annulata in north of Basrah province, AL-Qadisiya. The Journal of Veterinary Medical
Science 11(1): 54-62.

11. Al-Hosary AAT, Elsayed HK, Ahmed LS (2015) Oxidative stress and haematological profile in Theileria annulata clinically infected cattle before and after treatment. Assiut Veterinary Medical Journal 61(144): 123-129.

12. Goyal P, Chahar A, Tanwar RK, Fakhruddin (2017) Clinico-physiological and haematological studies in cattle-calves naturally infected with Theileria annulata by nested PCR. Bulletin of Environment Pharmacology and Life Sciences 6(3): 39-44.

13. Padhiyar AP, Jadhav KM, Patel Bharatkumar K, Chauhan HC, Patel Kirit B (2016) Evaluation of molecular biology for identification Theileriosis disease in crossbred cattle. International Journal of Agriculture Sciences 8 (63): 3575-3577.

14. Zahid IA, Latif M, Baloch KB (2005) Incidence and treatment of theileriasis and babesiasis. Pakistan Veterinary Journal 25(3): 137-139.

15. Kumar, Monika N, Parmar KP (2019) Bovine tropical theileriosis in Tharparkar calves and its therapeutic management. International Journal of Current Microbiology and Applied Sciences 8(3): 983-986.

16. Singh J, Gill JS, Kwatra MS, Sharma KK (1993) Treatment of theileriosis in crossbred cattle in the Punjab. Tropical Animal Health and Production 25(2): 75-78.

17. Modi, DV, Bhadesiya CM, Mandali GC (2015) Haematobiochemical Changes in Cross-bred Cattle Infected with Theileria annulata in Banaskantha District of Gujarat. International Journal of Scientific and Research Publications 5(1): 1-4. 